\title{
Subversion of human intestinal mucosa innate immunity by a Crohn's disease-associated E. coli
}

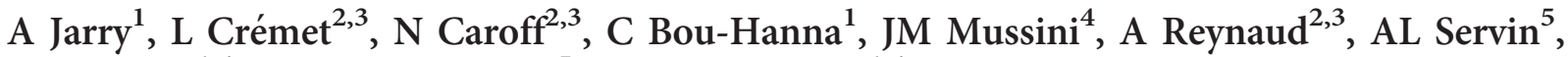 \\ JF Mosnier ${ }^{1,4}, \mathrm{~V}$ Liévin-Le Moal $^{5}$ and CL Laboisse ${ }^{1,4}$
}

Adherent-invasive Escherichia coli (AIEC), associated with Crohn's disease, are likely candidate contributory factors in the disease. However, signaling pathways involved in human intestinal mucosa innate host response to AIEC remain unknown. Here we use a 3D model of human intestinal mucosa explant culture to explore the effects of the AIEC strain LF82 on two innate immunity platforms, i.e., the inflammasome through evaluation of caspase-1 status, and $\mathrm{NF}$ KB signaling. We showed that LF82 bacteria enter and survive within a few intestinal epithelial cells and macrophages, without altering the mucosa overall architecture. Although 4-h infection with a Salmonella strain caused crypt disorganization, caspase-1 activation, and mature IL-18 production, LF82 bacteria were unable to activate caspase-1 and induce IL-18 production. In parallel, LF82 bacteria activated NFKB signaling in epithelial cells through

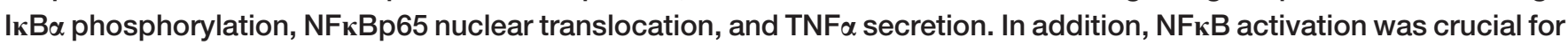
the maintenance of epithelial homeostasis upon LF82 infection. In conclusion, here we decipher at the whole-mucosa level the mechanisms of the LF82-induced subversion of innate immunity that, by maintaining host cell integrity, ensure intracellular bacteria survival.

\section{INTRODUCTION}

Intestinal innate immunity can be operationally defined as the set of mechanisms, present within intestinal mucosa-resident cells such as epithelial cells and underlying macrophages, that are rapidly available to keep potential pathogens under control and thus maintain mucosa homeostasis. Two multiprotein platforms are involved in innate immunity of the intestinal mucosa, the inflammasome, and the nuclear factor kappa-lightchain-enhancer of activated B cells (NFKB) pathways.

The inflammasome is a caspase- 1 activating platform, which promotes cleavage of pro-interleukin (IL)- $1 \beta$ and pro-IL18 in their mature active forms. ${ }^{1}$ These pro-inflammatory cytokines, mainly IL-18 known as an interferon (IFN)- $\gamma$-inducing cytokine, can elicit a subsequent Th1 response. The intestinal mucosa is a reservoir of procaspase- 1 and pro-IL-18, present in the main cellular compartment, the epithelial cells, and also in some lamina propria macrophages, ${ }^{2-4}$ that can be rapidly activated under various stress conditions. ${ }^{5}$ Caspase- 1 activation is crucial for mounting an acute inflammatory reaction, which is the primary defense against pathogenic bacteria. In addition, caspase- 1 induces an inflammatory cell death called pyroptosis, a likely mechanism of bacterial clearance. ${ }^{6,7}$

The NFאB pathway is another multi-protein platform involved in innate immunity. In an inactivated state, NFкB is located in the cytosol, complexed with the inhibitory protein $\mathrm{I} \kappa \mathrm{B} \alpha$. Upon various stimuli, $\mathrm{I} \kappa \mathrm{B} \alpha$ is phosphorylated, which results in ubiquitinylation, dissociation of $I \kappa B \alpha$ from $N F \kappa B$, and $I \kappa B \alpha$ degradation in the proteasome. The activated $N F \kappa B$ is then translocated into the nucleus, where it induces target gene transcription, including pro-inflammatory cytokines such as TNF $\alpha .{ }^{8}$ NFKB can be activated by a variety of microbial products and is involved in bacterial clearance. ${ }^{9,10}$ In addition, depending on the context, $\mathrm{NF \kappa B}$ activation has a pro-apoptotic or anti-apoptotic effect in intestinal epithelial cells. ${ }^{11,12}$

The activation of these two platforms (i) shapes the interactions between pathogens and mucosa-resident cells of the host, and also (ii) results in the mobilization and recruitment of circulating leukocytes at the site of infection.

${ }^{1}$ EA 4273 Biometadys, Université de Nantes, Faculté de Médecine, Nantes, France. ${ }^{2}$ UPRES EA3826 Université de Nantes, Faculté de Médecine, Nantes, France. ${ }^{3}$ CHU Nantes, Service de Bactériologie-Hygiène, Nantes, France. ${ }^{4} \mathrm{CHU}$ Nantes, Service d'Anatomie et Cytologie Pathologiques, Nantes, France and ${ }^{5} \mathrm{CNRS}$ UMR8076, Université Paris-Sud, Faculté de Pharmacie, Chatenay-Malabry, France. Correspondence: A Jarry or C Laboisse (ajarry@univ-nantes.fr or christian.laboisse@chu-nantes.fr) 
These two events are tightly interconnected, and this makes it difficult to delineate the very first steps, i.e., the interaction between pathogens and intestinal mucosa innate immune cells. To study such an interaction implies to have a model system with the following requirements: maintain the $3 \mathrm{D}$ architecture of the intestinal mucosa and the cellular interactions between resident innate immune cells; maintain the major recognition pathways of bacterial structures; be isolated from the vascular compartment and thus devoid of recruited immune cells from peripheral blood. The recently developed ex vivo explant cultures of human intestinal mucosa fulfils these requirements. ${ }^{13}$ This ex vivo model is a powerful system to delineate the signaling pathways that either maintain human intestinal mucosa homeostasis or initiate an inflammatory response. ${ }^{5,13,14}$

Crohn's disease (CD) is a multifactorial inflammatory bowel disease caused by a combination of factors, including genetics, the intestinal microbiota, environmental factors, and enteric pathogens. Adherent-invasive Escherichia coli (AIEC) strains, representing a new pathotype of enteric pathogenic E. coli, ${ }^{15}$ have been recently isolated from intestinal mucosa of $\mathrm{CD}$ patients. ${ }^{16,17}$ In fact, AIEC strains can represent $>50 \%$ of the total bacteria present in the mucosa of CD patients and are also found at low levels in the mucosa of healthy individuals. ${ }^{16-19}$ In addition, in reductionist in vitro models, the prototype AIEC strain LF82 has been reported to colonize human colonic cancer cell lines, as well as macrophage cell lines, often of murine origin. ${ }^{20-23}$ The signaling pathways involved in innate host defense against AIEC, after AIEC bacteria interacted with intestinal mucosa-resident cells, remain largely unknown. The use of murine cell lines to study the response of macrophages to AIEC infection ${ }^{24}$ may be misleading because of the biological differences between murine and human macrophages. ${ }^{25}$ In this context, a primary challenge in understanding how AIEC could remain silent and/or contribute to a chronic inflammatory bowel disease is to delineate the way they colonize the human normal intestinal barrier and interact with the innate immune system.

The aim of this work was to delineate the early interactions of AIEC prototype strain LF82 with two platforms involved in intestinal innate immunity of mucosa-resident cells, i.e., the inflammasome and the NFKB pathway. To do this, we used human intestinal mucosa explant cultures, an ex vivo model that approaches at its best the in vivo situation. Our findings provide the first demonstration that an AIEC strain is able to subvert the innate immune response in human intestinal mucosa-resident cells.

\section{RESULTS}

\section{The AIEC strain LF82 is adherent to and invasive in intestinal epithelial cells and lamina propria mononuclear cells}

The ex vivo model of explant cultures of human normal colonic mucosa was used to examine the interactions of the AIEC strain LF82 with human colonic mucosa cells. To this end, explant cultures, left to stabilize overnight in culture medium with antibiotics, were co-incubated with LF82-green fluorescent protein (GFP) $\left(10^{8}\right.$ or $10^{9}$ bacteria per explant) without antibiotics for $4 \mathrm{~h}$. As shown in Figure 1a, LF82 bacteria, detected by immunoperoxidase using an anti-GFP antibody on paraffin sections, were found adhering to the apical pole of a few epithelial cells of the surface and crypt base, scattered or sometimes focally clustered. Positive immunostaining was also observed within some epithelial cells. GFP-expressing bacteria were also present in the lamina propria, in the subepithelial area where numerous $\mathrm{CD} 68+$ macrophages are present (Figure 1a, right). Detection of GFP bacteria by immunofluorescence on frozen sections confirmed the presence of bacteria in a few epithelial cells and in lamina propria mononuclear cells resembling macrophages (Figure 1b). Cytospin preparations of isolated cells obtained after collagenase digestion confirmed the presence of GFP bacteria within large cells resembling macrophages (Figure 1c). Giemsa stain on cytospins showed the occurrence of bacteria localizing intracellularly in vacuoles (Figure 1d). Double immunofluorescence staining confirmed the presence of LF82-GFP in CD68 + lamina propria macrophages (Figure 1e). Transmission electron microscopy confirmed the presence of internalized bacteria within intracellular membrane-bounded vacuoles (Figure 2) and in lamina propria phagocytes.

To get insight into the mechanism of entry of LF82 into mucosal cells, especially epithelial cells, we examined the expression of CEACAM6, a molecule that contributes to AIECmucosal cell interactions in inflammatory conditions. ${ }^{26} \mathrm{We}$ assessed by immunohistochemistry the expression of CEACAM6 in colonic mucosa explants, before culture and after a 24-h culture, in seven colonic samples. Before culture, CEACAM6 expression was restricted to surface epithelial cells (apical membrane staining) and, inconstantly, to the epithelial cells of the upper third of the crypt (Figure 3). Interestingly, after a 24-h explant culture, for all cases studied, CEACAM6 expression by surface epithelial cells was higher than before culture and extended to the whole colonic crypt (Figure 3). This staining pattern was unchanged in LF82-infected explant cultures.

\section{Intracellular survival of LF82 in colonic mucosa cells}

To confirm that LF82 was able to enter mucosa cells, a quantitative assessment of live bacteria was set up to count only intracellular bacteria and discard extracellular bacteria that can be trapped in the mucus. After the 4-h incubation with LF82, the mucosa explants were washed and subjected to collagenase treatment for $1 \mathrm{~h}$ in the presence of gentamicin $\left(100 \mu \mathrm{g} \mathrm{ml}^{-1}\right)$ to kill extracellular bacteria. Mucosa cells were then filtered on a cell strainer and washed in PBS. The cell pellet was lysed with $0.1 \%$ Triton X-100, plated on solid medium plates, and colonies formed were counted. As shown in Table 1, measurable colonyforming units (CFUs) ranging from 123 to $442 \mathrm{CFU}$ per $10^{7}$ mucosa cells (mean \pm s.e.m.: $254 \pm 96$ ) were observed, with an inter-individual variability. Importantly, no CFUs were observed in the "sterility controls," performed in the spent medium of the last wash before performing cell lysates, as well 

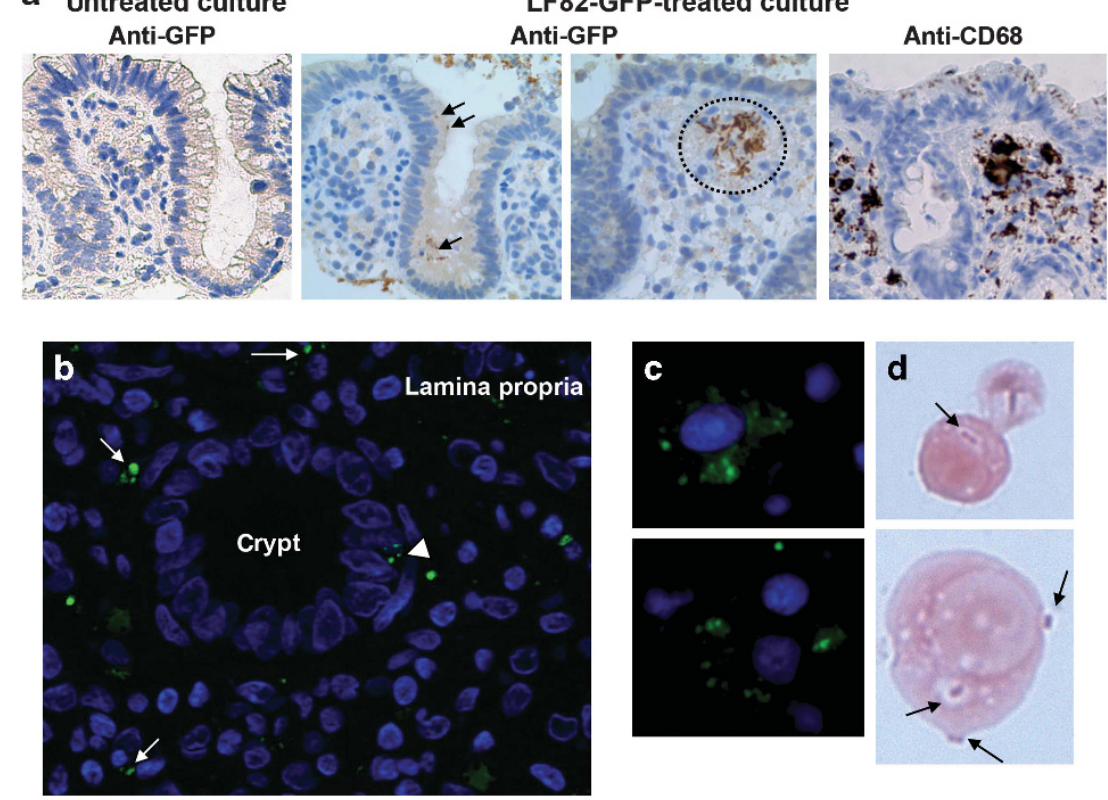

e
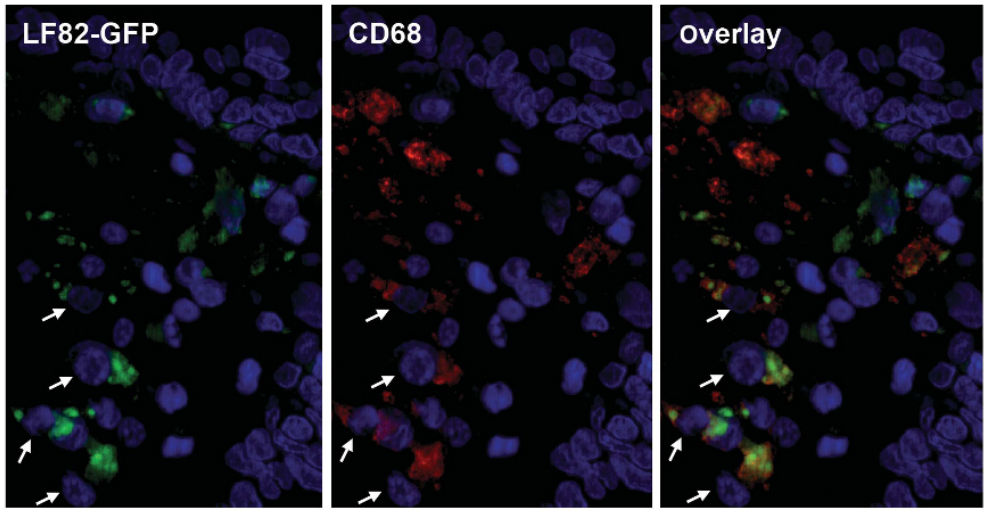

Figure 1 AIEC LF82 invades human normal colonic mucosa innate immune-resident cells. Detection of LF82-GFP in explant cultures of human normal colonic mucosa after a 4-h incubation. (a) Immunoperoxidase staining on paraffin sections with anti-GFP antibodies shows the presence of GFP-bacteria (brown) at the apical pole of the epithelial lining (arrows) and also in the lamina propria (dotted circle), in the subepithelial area where numerous CD68 + macrophages are present (right). Untreated cultures (not infected with LF82-GFP) served as a negative control for GFP staining. (b, c)

Immunofluorescence detection of GFP on frozen sections (b) and on cytospin preparations of cells isolated from the colonic mucosa explants after collagenase digestion (c) confirms the presence of GFP bacteria (green) within a few epithelial cells (arrowhead) and lamina propria mononuclear cells resembling macrophages (arrows). (d) Gram stain on cytospin preparations shows bacteria within vacuoles (arrows). (e) CD68 immunofluorescence staining shows the presence of LF82 (green) in some CD68 + lamina propria macrophages (red, arrows). Nuclei appear in blue.

as in the uninfected controls. Altogether, these results indicate that LF82 is able to survive intracellularly after entry within colonic mucosa cells.

\section{LF82 does not alter the overall architecture of the colonic mucosa}

We next explored the consequences of the adherence/invasion of LF82 on the mucosal architecture. As shown in Figure 4, LF82 $\left(10^{8}\right.$ or $10^{9}$ bacteria per explant) did not elicit major disruption of the colonic mucosa after a 4 -h incubation. The crypt height was similar to that of control cultures. The surface epithelium of the LF82-infected mucosa underwent higher apoptosis than in control cultures, as assessed by M30 immunostaining (Figure 4, middle). However, very few apoptotic cells were noted at the crypt base. In the same culture conditions, we verified that Salmonella enterica serovar Typhimurium (S. Typhimurium), ${ }^{27}$ a highly invasive enteric pathogen, was able to induce mucosa disruption. Indeed, incubation of colonic mucosa explant cultures with $S$. Typhimurium $\left(10^{8}\right.$ bacteria per explant for $\left.4 \mathrm{~h}\right)$ induced a marked destruction of the surface epithelium and crypt base, leading to complete destruction of numerous crypts, through massive apoptosis, as assessed by M30 immunostaining (Figure 4, right).

\section{LF82 elicits NFкB activation, which is preceded by ІкB $\alpha$ phosphorylation}

We next examined whether LF82 was able to interfere with the $\mathrm{NF \kappa B}$ signaling pathway, whose activation is known to have a cytoprotective effect on intestinal epithelial cells under various 

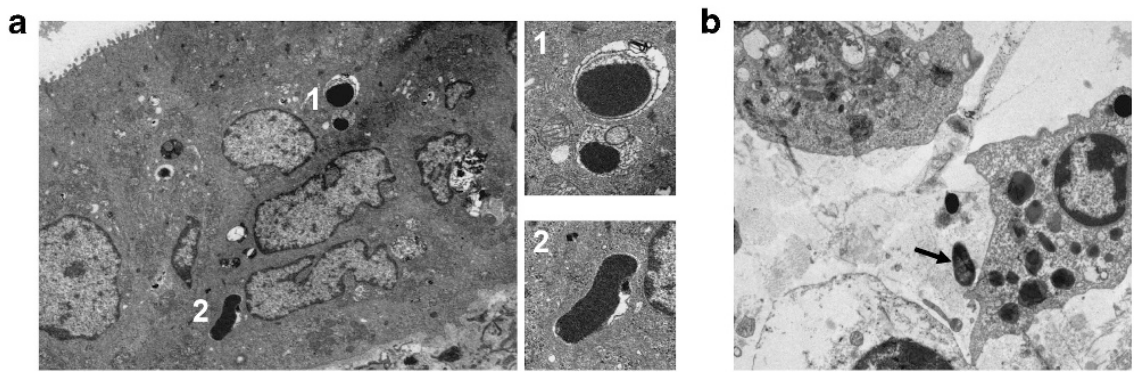

Figure 2 Electron microscopy examination of the LF82-infected cells. Transmission electron microscopy of a 4-h LF82-infected mucosa explant culture shows bacteria within membrane-bounded vacuoles in some intestinal epithelial cells (a; inset 1 and 2: higher magnification) and lamina propria phagocytes (b, arrows: bacteria on contact with and within a phagocyte).

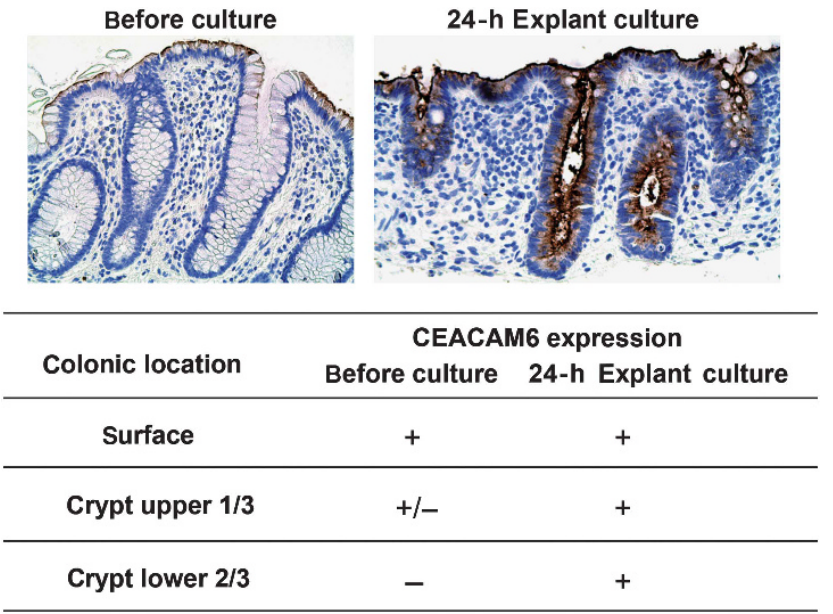

Figure 3 CEACAM6 expression in human colonic crypts after a 24-h explant culture. Immunoperoxidase staining on paraffin sections with antiCEACAM6 antibodies shows a positive staining of surface epithelial cells before culture (left) and an extension of the staining all along the crypt after a $24 \mathrm{~h}$ explant culture (right). The table shows a three tier evaluation of CEACAM6-positive epithelial cells along the colonic crypt ( - : no staining; $+/-$ : heterogeneous staining; + : positive staining). Representative of seven independent explant cultures.

stress conditions. We first examined the nuclear translocation of NFKB p65 in colonic crypts of LF82-treated explants, using immunohistochemistry on paraffin sections after a 4-h incubation with LF82 ( $10^{9}$ bacteria per explant). As shown in Figure 5a (middle), the epithelial cells of all crypts showed numerous NFKB p65-positive nuclei $(73 \% \pm 9 \%, n=4$ experiments, range 40-83\%) (Figure 5a, histogram). In control uninfected cultures, and in explant cultures infected with a commensal E. coli strain (MG1655), NFkB p65 was observed only in the cytoplasm of epithelial cells (Figure 5a, left and right). We next examined whether LF82-induced NFKB activation was preceded by an I $\mathrm{B} \alpha$ phosphorylation on ser3236 , a key step necessary for I $\mathrm{I} B \alpha$ degradation and subsequent $\mathrm{NF \kappa B}$ translocation into the nucleus. Colonic mucosa explant cultures were incubated in the absence or in the presence of LF82 for $1 \mathrm{~h}$. The explants were then collected in lysis buffer, and subjected to SDS-PAGE and immunoblot analysis with antibodies against phospho-IKB $\alpha$. As shown in Figure 5b, LF82 slightly induced IкB $\alpha$ phosphorylation, which was not detected
Table 1 LF82 is able to survive in the human normal colonic mucosa-infected cells

\begin{tabular}{cc} 
Sterility controls & Cell lysates plated on \\
$\mathrm{CFU} / 10^{7}$ cells & solid medium-CFU/10 $70^{7}$ cells \\
\hline
\end{tabular}

Experience 1

Control

0

0

LF82

0

$197 \pm 84^{a}$

Experience 2

Control

0

0

LF82

0

$442 \pm 59^{\mathrm{a}}$

\section{Experience 3}

Control

0

0

LF82

0

$123 \pm 33^{\mathrm{a}}$

Abbreviation: CFU, colony-forming unit. Human normal colonic mucosa explant cultures, infected or not with LF82 $\left(10^{9}\right.$ bacteria per explant for $\left.4 \mathrm{~h}\right)$, were then incubated with collagenase in the presence of gentamicin to kill extracellular bacteria (gentamycin protection assay), as described in Methods.

aMean \pm s.e.m. of three cell preparations from three explants pooled in the medium containing collagenase (see Methods).

in control cultures. Interestingly, pretreatment with ALLN (100 $\mu \mathrm{g} \mathrm{ml}^{-1}$ for $40 \mathrm{~min}$ ), a proteasome inhibitor that blocks degradation of I $\mathrm{I} B \alpha$, before LF82 infection, greatly enhanced $\mathrm{I} \kappa \mathrm{B} \alpha$ phosphorylation (Figure $5 \mathbf{b}$ ).

\section{Blockade of LF82-induced NFKB activation leads to a massive epithelial cell apoptosis}

To examine the role of LF82-induced NFKB activation, we pretreated the mucosa explants cultures with $\mathrm{SN} 50$, a cell permeable inhibitor peptide that blocks NFkB translocation, before a 4-h infection with LF82. Preliminary experiments showed that SN50 was effective in the explant cultures, as its pre-treatment before LF82 infection led to a significant decrease (by 70\%) in the number of NFKB p65-positive nuclei $(25 \% \pm 5 \%$ NFkB p65-positive nuclei, $n=4)$ compared with explant cultures incubated with LF82 in the absence of SN50 $(78 \% \pm 9 \% \mathrm{NF \kappa B}$ p65-positive nuclei, $n=4)$. As shown in Figure 6a, a pretreatment with SN50 led to a severe destruction of both surface and crypt epithelium in LF82-infected explants. 

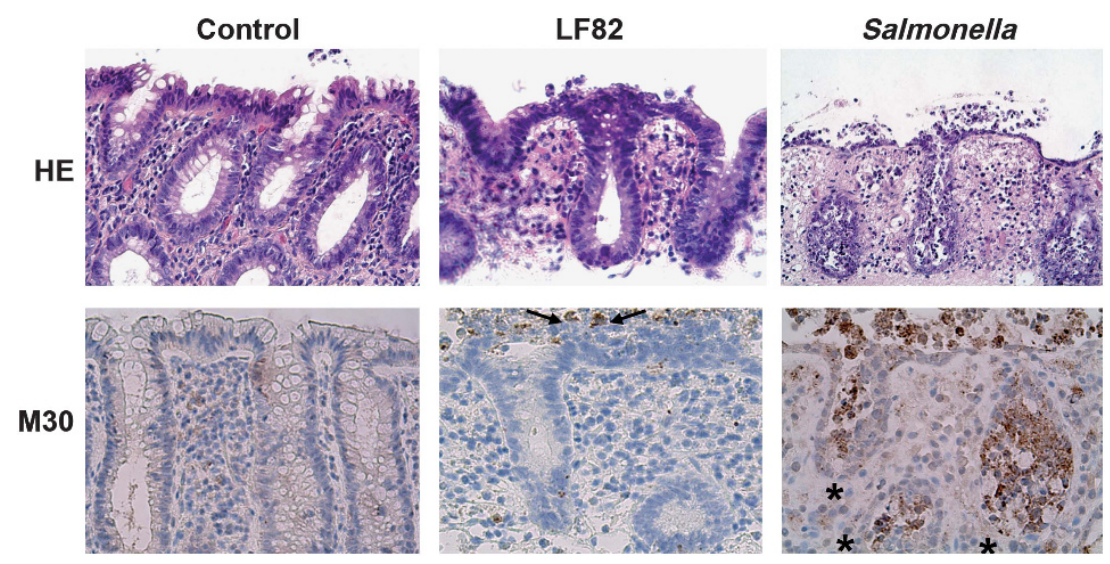

Figure 4 AIEC LF82 does not induce major disruption of human normal colonic crypts. Human normal colonic mucosa explant cultures were treated or not for $4 \mathrm{~h}$ with LF82 ( $10^{9}$ bacteria per explant) or with $S$. Typhimurium (10 ${ }^{8}$ bacteria per explant). Hematoxylin-eosin (HE) standard staining and immunoperoxidase staining with the M30 antibody specific for apoptotic epithelial cells. LF82 does not alter the colonic mucosa architecture and induces only low epithelial apoptosis, mainly in the surface epithelium (brown cells, arrows), but not at the crypt base. By contrast, $S$. Typhimurium completely disrupts epithelial crypts including crypt base containing many M30-positive cells (asterisks).

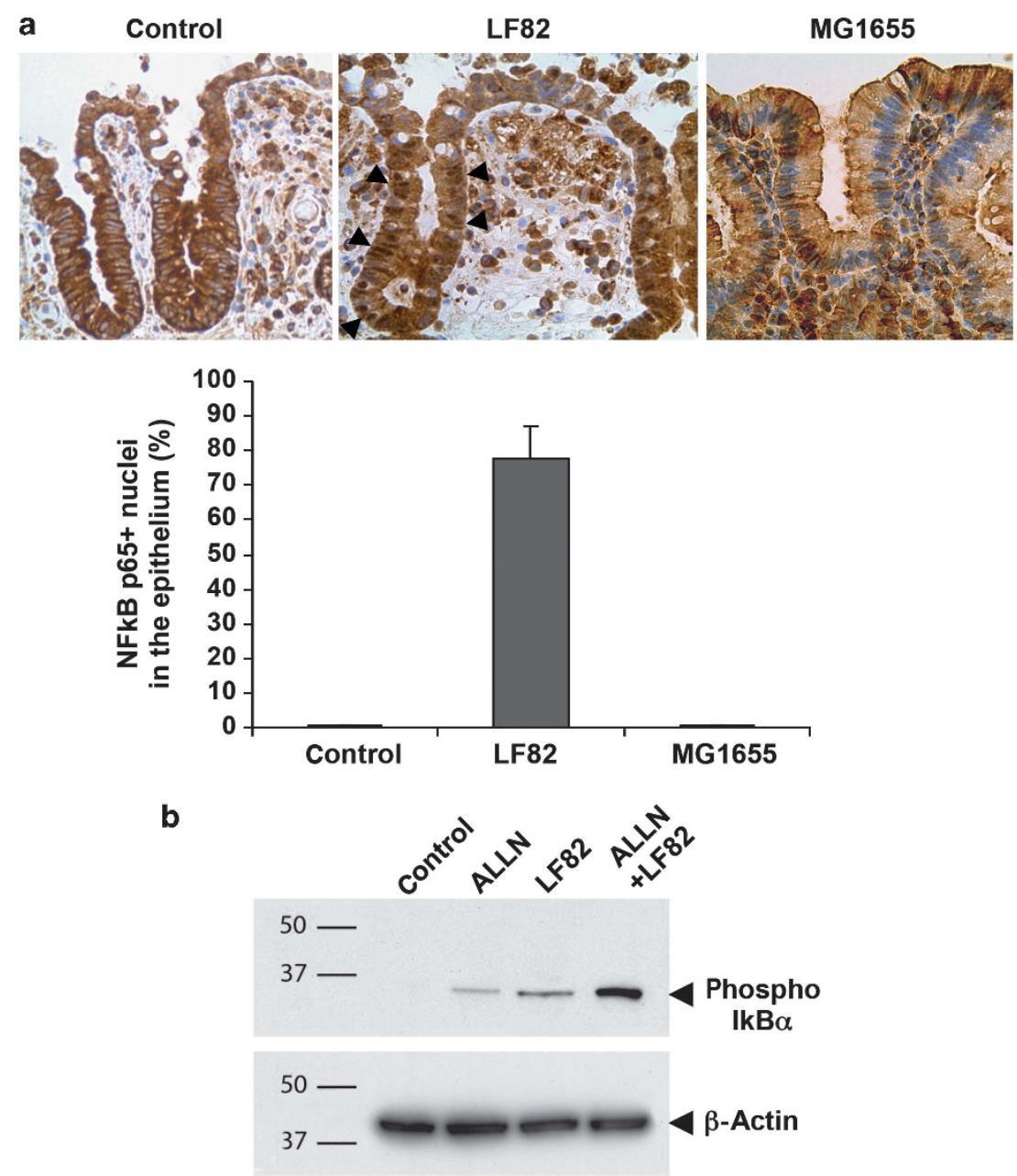

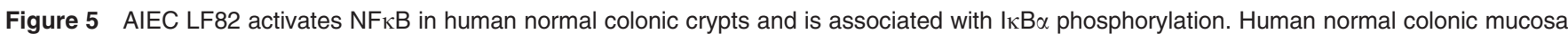
explant cultures were treated or not for $4 \mathrm{~h}$ with LF82 ( $10^{9}$ bacteria per explant), or with the commensal E. coli strain MG1655. (a) Immunoperoxidase staining with an antibody directed to NFKB p65 subunit. LF82 (middle), but not MG1655 (right), elicits a cytoplasmic-to-nuclear translocation of NFKB p65 (arrowheads) in the crypt epithelial cells. The histogram indicates the percentage of NFKB p65-positive nuclei in crypt epithelial cells of LF82-infected explant cultures compared with control untreated cultures, or with MG1655-infected explant cultures. Mean \pm s.e.m. of four experiments. (b) Immunoblot analysis of phospho-IKB $\alpha$ (ser 32-36) in explant cultures treated or not for $1 \mathrm{~h}$ with LF82 (10 ${ }^{9}$ bacteria per explant), with or without pretreatment with the proteasome inhibitor ALLN (100 $\mathrm{gg} \mathrm{ml}^{-1}$ for $\left.40 \mathrm{~min}\right)$. Equal protein load was evaluated by $\beta$-actin. Representative of three independent experiments. 
a
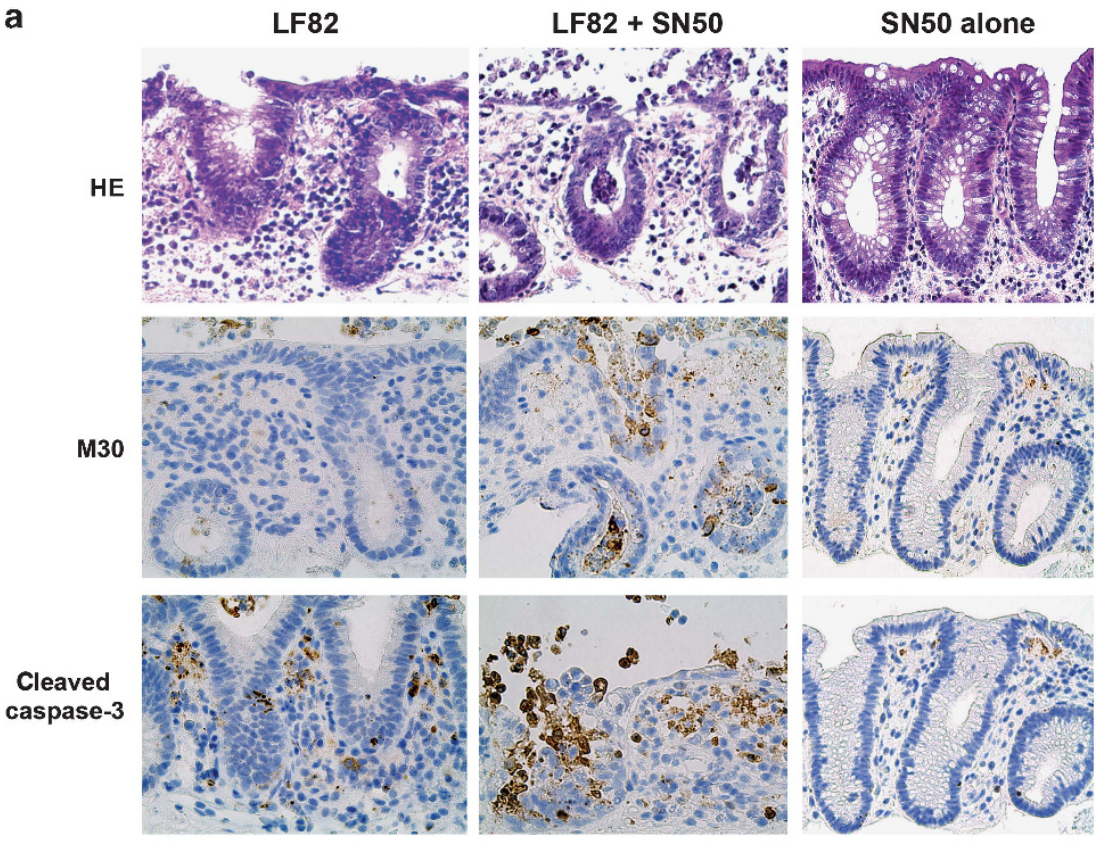

b
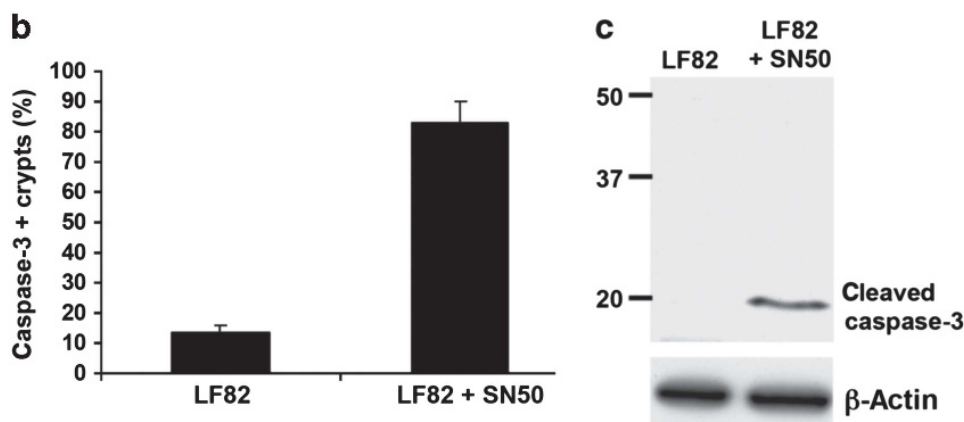

Figure 6 Blockade of NFKB activation by AIEC LF82 induces massive epithelial apoptosis. Human normal colonic mucosa explant cultures were treated or not for $4 \mathrm{~h}$ with LF82 ( $10^{9}$ bacteria per explant), with or without pretreatment with the NFKB nuclear translocation inhibitor SN50 (100 $\mu$ g ml ${ }^{-1}$ for $40 \mathrm{~min}$ ). (a) Hematoxylin-eosin (HE) standard staining, and immunoperoxidase staining with $\mathrm{M} 30$ and cleaved caspase-3 antibodies. In the presence of LF82, SN50 induces epithelial disruption and a massive increase in M30 and cleaved caspase-3-positive epithelial cells (brown cells; nuclei counterstained in blue). SN50 alone has no effect on crypt viability (right). (b) Quantitative evaluation of caspase-3-positive crypts (containing more than $30 \%$ positive epithelial cells) in LF82-infected explants in the presence or absence of SN50. Mean \pm s.e.m. of three independent experiments. (c) Immunoblot analysis of cleaved caspase-3 in lysates from LF82-treated explants with or without SN50. Representative of three independent experiments.

These disrupted epithelial cells underwent an apoptotic process, as shown by their strong immunoreactivity with M30 and active caspase-3 antibodies (Figure 6a, middle). Of note, SN50 treatment did not induce epithelial damage in uninfected control cultures (Figure 6a, right). Evaluation of the percentage of caspase-3-positive crypts (containing more than $30 \%$ caspase-3-positive epithelial cells) showed a significant increase in apoptotic crypts in LF82-infected explants pretreated with SN50 compared with cultures in the absence of SN50 $(P=0.001)$ (Figure $6 \mathbf{b})$. Immunoblot analysis of the LF82-infected explants pretreated with SN50 shows that the anti-caspase- 3 antibody recognizes a single band of low MW corresponding to the active form of caspase- 3 and does not recognize the high MW precursor form of caspase- 3 (Figure 6c).

\section{LF82 does not trigger the inflammasome pathway}

We next addressed whether LF82 could activate the inflammasome pathway, a multiprotein complex that mediates the activation of caspase-1, which promotes maturation/secretion of the proinflammatory cytokines IL-1 $\beta$ and IL-18. We examined the effect of LF82 on the inflammasome pathway by measuring (i) caspase-1 activity (cleavage of a selective substrate detected by spectrophotometry) and (ii) IL-18 secretion by enzyme-linked immunosorbent assay (ELISA). As shown in Figure 5a, no significant increase in caspase-1 activity was found in LF82-infected explant cultures (4-h incubation) compared with control cultures. By contrast, $S$. Typhimurium, known to activate the inflammasome pathway and thus used as a positive control, induced caspase- 1 activity (sixfold control value) (Figure 7a). Accordingly, LF82 did not 

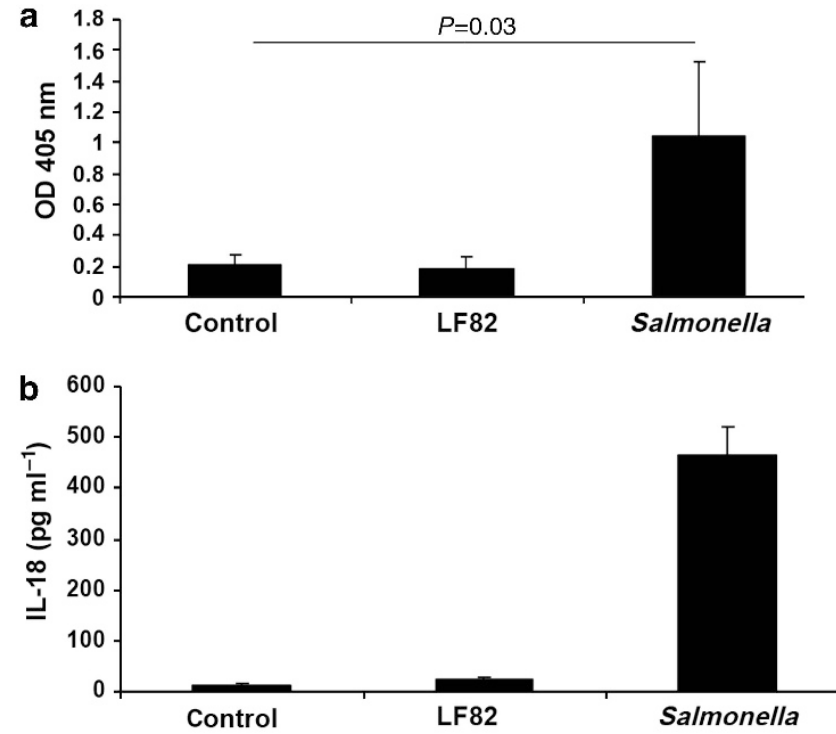

Figure 7 AIEC LF82 does not activate the inflammasome pathway. Human normal colonic mucosa explant cultures were treated or not for $4 \mathrm{~h}$ with LF82 ( $10^{9}$ bacteria per explant) or with $S$. Typhimurium $\left(10^{8}\right.$ bacteria per explant), used as a positive control. (a) Caspase-1 activity detected in cell lysates, by colorimetric assay with a specific substrate (YVAD-pNA). Absorbance at $405 \mathrm{~nm}$. Mean \pm s.e.m. of three different experiments. (b) Measurement of IL-18 secretion by ELISA in the supernatants of the 4-h explant cultures. Mean \pm s.e.m. of five independent experiments with at least four explant cultures per condition.

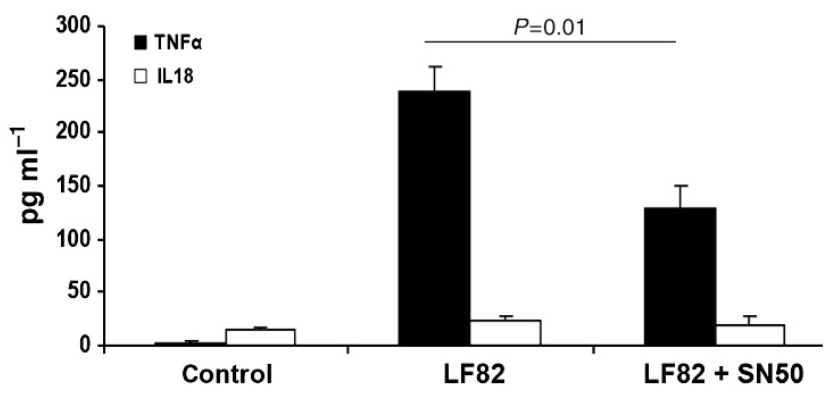

Figure 8 NFkB does not interfere with the inflammasome status in LF82treated mucosa explant cultures. Human normal colonic mucosa explant cultures were treated or not for $4 \mathrm{~h}$ with LF82 $\left(10^{9}\right.$ bacteria per explant) with or without pretreatment with SN50. TNF $\alpha$ and IL-18 secretion were assayed in the supernatants by ELISA. Mean \pm s.e.m. of five independent experiments with at least four explant cultures per condition.

induce mature IL-18 secretion in 4-h mucosa explant cultures, whereas $S$. Typhimurium led to a strong release of IL-18 in 4-h mucosa explant cultures (Figure $7 \mathbf{b}$ ).

\section{NFKB does not interfere with the inflammasome status of mucosal cells}

We next examined the inflammasome response in relation with NFкB activation. As shown in Figure 8, LF82 led to a strong $\mathrm{TNF} \alpha$ response in 4-h mucosa explant cultures, which was significantly decreased by the inhibitor peptide SN50. Interestingly, SN50 did not induce any IL-18 response in LF82-treated mucosa explants. Altogether, these results show that LF82 induced an NFאB-dependent TNF $\alpha$ production and that $\mathrm{NF \kappa B}$ did not interfere with the inflammasome status of mucosal cells.

\section{DISCUSSION}

Up to now, only reductionist in vitro model systems, mainly colonic cancer cell lines, have been used to study the early interactions of pathogens such as AIEC with intestinal epithelial cells. ${ }^{28}$ The main end points studied concerned mechanisms involved in adherence/invasion of epithelial cells, epithelial barrier disruption, and, in very few studies, production of chemokines such as IL-8. However, the use of reductionist models is not suitable for deciphering the interactions of bacteria with epithelial cell innate immune platforms as well as their consequences. Indeed, caspase-1/ inflammasome has been shown to be downregulated or absent in many colonic cancer cell lines. ${ }^{2,3}$ In addition, the NFKB signaling pathway has been shown to be altered in oncogenesis and to participate in deregulated cancer cell growth and apoptosis. ${ }^{29,30}$ Here, we provide the first demonstration that explant culture of human normal colonic mucosa, an integrated model system, is the most suitable method to decipher the interactions between pathogens and innate immune system signaling pathways in relation with intestinal mucosa homeostasis.

The main objective of this work was to study the cross talk between the AIEC-prototype strain LF82 and the innate immunity platforms-inflammasome and NFKB-of the human normal intestinal mucosa resident cells, which has never been explored yet. As our experimental system aimed at examining the effect of LF82 on innate mucosal immunity irrespective of the polarity of entry into mucosa-resident cells, we used a simple approach that did not require the use of a polarized exposition to bacteria. ${ }^{31} \mathrm{We}$ first studied the interaction of LF82 with epithelial and immune cells by morphological methods (immunohistochemistry, immunofluorescence, electron microscopy) and monitored the number of bacteria entering cells by setting up a gentamicin protection assay after collagenase digestion of the infected explants to kill extracellular bacteria. We were able to show that LF82 can enter a few epithelial cells and macrophages, which are both mucosaand innate immune-resident cells. Interestingly, LF82 did not alter the overall architecture of the mucosa and can survive within resident mucosa cells. The number of intracellular bacteria found after the gentamicin protection assay was variable among individuals suggesting that factors, still unknown, may regulate bacterial entry in mucosal-resident cells. This observation led us to examine the expression of CEACAM6, which acts as a receptor for AIEC, ${ }^{26}$ in our explant culture conditions. In fact, before culture, the gradient of CEACAM6 expression we observed along the colonic cryptsurface axis is in line with the pattern of CEACAM6 (previously called NCA/CD66c) expression described by Hammarstrom. ${ }^{32}$ Interestingly, after a 24 -h explant culture, this gradient was replaced by a homogeneous and strong CEACAM6 expression all along the colonic crypt. Together, our results show that the 
human colonic explant culture system, in addition to maintaining its morphological integrity over a $24 \mathrm{~h}$ culture, maintains the receptors for LF82.

We show here that LF82 does not activate caspase- 1 and does not elicit mature IL-18 release by resident innate immune cells, after a short $(4 \mathrm{~h})$ or longer $(9 \mathrm{~h})$ infection time. We verified that the inflammasome is functional in our model system, since, as expected, $S$. Typhimurium activates caspase-1, leading to IL-18 release in the same experimental conditions. These results are in line with the severe acute enterocolitis observed in vivo in Salmonella infections. ${ }^{33}$ Our finding of absence of inflammasome activation upon LF82 infection is the first demonstration that AIEC have evolved, despite their expression of flagellin, ${ }^{34}$ a way of escaping recognition by the inflammasome sensors of human normal mucosa-resident cells. It can be hypothetized that, upon LF82 infection, the absence of caspase-1 activation and pyroptosis could represent an evasion strategy, and could favor bacterial load and intracellular bacteria survival, without inducing an acute inflammatory response.

In parallel, we show here that LF82 activates NFKB, preceded by $I \kappa B \alpha$ phosphorylation, necessary for I $\mathrm{K} \beta \alpha$ degradation in the proteasome. I $B \alpha$ degradation could result from the modulation of the ubiquitin proteasome system functioning by LF82 bacteria. ${ }^{35}$ In addition, our finding that a commensal $E$. coli strain was unable to activate NFKB in mucosa explants suggests that NFKB activation could be specific of LF82. The LF82-induced NFKB activation could be accounted for by different mechanisms such as contact, intracellular entry, or even production of soluble factors. Our results suggest that this LF82-induced NFKB activation, occurring mainly in epithelial cells, could be a protective innate immune mechanism. To test this hypothesis, and assess the local effects of NFKB on the intestinal mucosa resident cells, we treated the mucosa explants with $\mathrm{NF \kappa B}$ inhibitors. Interestingly, we show here that blockade of $\mathrm{NF \kappa B}$ translocation by the inhibitory peptide SN50 in LF82-infected explant cultures leads to a massive disruption of epithelial crypts via apoptosis. Thus, LF82induced activation of $\mathrm{NF \kappa B}$ has a protective role in intestinal mucosa-resident cells, especially in epithelial cells. Altogether, these results strongly suggest that LF82, by activating NFKB and thus preventing apoptosis, subverts the innate immune response of intestinal mucosa-resident cells. In addition, our demonstration of a NFKB-dependent TNF $\alpha$ release upon LF82 infection suggests an additional mechanism of subversion as TNF $\alpha$ has been shown to facilitate intracellular bacteria survival and bacteria growth in a macrophage cell line. ${ }^{23}$

Altogether, we have delineated, using a 3-D model of human intestinal mucosa explant cultures, two mechanisms allowing the CD-associated AIEC strain LF82 to subvert the innate immune system of the human intestinal mucosa-resident cells, one by escaping the inflammasome activation and the other by activating the NFKB pathway. These two mechanisms are complementary as they ensure survival of LF82 bacteria within the intestinal mucosa. One can speculate that this association of LF82 with the human normal intestinal mucosa induces a shift in the regulatory mechanisms of mucosa homeostasis, which could lead to the onset of inflammatory bowel disease in susceptible individuals.

\section{METHODS}

Human colonic mucosa explant cultures. Human normal colon was obtained from 22 patients undergoing surgery for colon carcinoma (14 men and 8 women; mean age $69 \pm 3.5$ years; range $41-89$ years; proximal colon $n=10$; distal colon $n=12$ ). The tissue fragments were processed according to the French guidelines for research on human tissues. Informed patient consent was obtained according to the French bioethics law. The mucosa was carefully stripped from the underlying compartment as previously described. ${ }^{13}$ Fragments of $40 \mathrm{mg}$ were maintained in culture overnight in RPMI-BSA 0.01\% supplemented with gentamicin $\left(70 \mu \mathrm{g} \mathrm{ml}^{-1}\right.$, Invitrogen, Life Technologies, SaintAubin, France) to get rid of commensal bacteria, and fungizone (1\%, Invitrogen), washed twice in RPMI, and then incubated for $4 \mathrm{~h}$ with or without bacterial cultures (LF82-GFP or S. Typhimurium) in $2 \mathrm{ml}$ culture medium without antibiotics (see below). The explants were maintained at $37^{\circ} \mathrm{C}$ in a $95 \%$ oxygen, $5 \%$ carbon dioxide humid atmosphere on a rocking platform at low speed. In each experiment, at least three explants were cultured for each condition. The supernatants were centrifuged and aliquots were stored at $-80^{\circ} \mathrm{C}$ for further analysis. Some tissue specimens were used for morphological analysis (optical microscopy or transmission electron microscopy) or immunohistochemical analysis, and the others were used for assessment of caspase- 1 activity or for immunoblot analysis (see below). We verified that a 24 -h culture did not modify colonic crypt length, epithelial cell polarity as assessed by immunofluorescence staining of the tight junction protein occludin, as well as the topography of the proliferative compartment (Supplementary Figure S1 online).

In a subset of experiments, before being challenged with bacteria, the explants were pretreated for $40 \mathrm{~min}$ with the following inhibitors: SN50 $\left(100 \mu \mathrm{g} \mathrm{ml}^{-1}\right.$, Enzo life Sciences, Villeurbanne, France), a synthetic peptide able to specifically inhibit the nuclear translocation of NFKB, or the proteasome inhibitor ALLN to block $\mathrm{I} \kappa \mathrm{B} \alpha(\mathrm{N}$-acetylleucyl-leucyl-norleucinal; $100 \mu \mathrm{g} \mathrm{ml}^{-1}$, Sigma, St Louis, MO). Then, the bacteria $\left(10^{9}\right.$ LF82-GFP per explant) were added and incubated for $4 \mathrm{~h}$ at $37^{\circ} \mathrm{C}$.

Bacterial strains and media. The prototype AIEC strain LF82-GFP was a generous gift from Pr Arlette Darfeuille-Michaud (UMR 1071 Inserm/Université Auvergne, Clermont-Ferrand, France). The strains were stored at $-20^{\circ} \mathrm{C}$ in cryotubes. Before the experiments, the bacteria were cultivated on TS agar at $37^{\circ} \mathrm{C}$ for $24 \mathrm{~h}$ after thawing. For each experiment, bacteria were subcultured in $\mathrm{LB}$ broth at $37^{\circ} \mathrm{C}$ for $18 \mathrm{~h}$ with shaking. The bacteria were then centrifuged for $10 \mathrm{~min}$ at $800 \mathrm{~g}$. The pellet was washed twice with sterile PBS, and the suspension was adjusted to $0.5 \times 10^{8}$ or $0.5 \times 10^{9}$ bacteria per milliliter, in culture medium (RPMI/BSA 0.01\% without antibiotics). The E. coli K12 MG1655 strain, used as a representative of commensal nonpathogenic E. coli, was grown as previously reported, ${ }^{13}$ and resuspended in culture medium at $0.5 \times 10^{8}$ bacteria per milliliter. $S$. Typhimurium was cultured overnight in LB broth at $37^{\circ} \mathrm{C}$ with shaking, and then subcultured at a 1:100 dilution for $3 \mathrm{~h}$. The bacteria were then washed with PBS and resuspended in culture medium at $0.5 \times 10^{8}$ bacteria per milliliter.

Gentamicin protection assay and assessment of intracellular live bacteria. To measure the number of intracellular live bacteria, and discard extracellular bacteria trapped in the mucus, the explants infected or not with LF82 for $4 \mathrm{~h}$ were washed with PBS, minced, and treated with type IV collagenase $\left(1 \mathrm{mg} \mathrm{ml}^{-1}\right.$, Sigma) for $1 \mathrm{~h}$ at $37^{\circ} \mathrm{C}$ with stirring, in the presence of $100 \mu \mathrm{g} \mathrm{ml}^{-1}$ gentamicin to kill extracellular bacteria. Three cultured explants of a given condition were pooled per tube of collagenase, to have enough cells. The cells were then filtered through a $100-\mu \mathrm{m}$ pore size cell strainer (BectonDickinson, San Diego, CA) and washed twice in PBS. A small aliquot of the cell suspension was counted using a hemocytometer. After 
centrifugation at low speed, the cell pellet was lysed in cold water containing $0.1 \%$ Triton X-100, for $10 \mathrm{~min}$ on ice. After vortexing, the lysates (undiluted or diluted 1:10) were plated on LB agar plates and incubated at $37^{\circ} \mathrm{C}$ for $24 \mathrm{~h}$. The numbers of intracellular bacteria were determined by counting CFUs. The results were expressed as CFU per $10^{7}$ cells. As a control, bacteria cultures were also performed in the spent medium of the last wash before performing cell lysates ("Sterility controls"). In some experiments, cytospin preparations of the cell suspension after collagenase digestion were performed and processed for immunoflorescence (see below) or Gram staining.

Transmission electron microscopy. After the 4-h incubation with LF82-GFP, the colonic mucosa explant cultures were rinsed in phosphate buffer $\mathrm{pH} 7.4$, fixed with $2.5 \%$ glutaraldehyde (Prolabo, France), postfixed in $2 \% \mathrm{OsO}_{4}$ (Agar, Oxford Instruments, Gometz la Ville, France) and embedded in Epon (Fluka, Sigma), using standard techniques. Ultrasections $(75 \mathrm{~nm})$ were contrasted with uranyl acetate and lead citrate, and examined using a transmission electron microscope (JEOL JEM1010, Croissy Sur Seine, France) equipped with a digital camera (GATAN Orius SC200, Evry, France).

Immunofluorescence and immunoperoxidase studies. Immunofluorescence was used to detect GFP-expressing LF82 on paraffin sections or paraformaldehyde-fixed frozen sections, or on cytospin preparations of isolated mucosa cells after collagenase digestion of the cultured explants. Immunofluorescence was also used to detect lamina propria macrophages using the anti-CD68 antibody (1:100, Dako, Les Ulis, France) and the Alexa fluor 568-conjugated goat anti-mouse antibodies (1:300, Invitrogen). Nuclei were stained with Dapi (1:1,000, Sigma). Sections were mounted using Prolong anti-fade medium (Invitrogen). The fluorescence was observed on a fluorescent microscope (Axiovert 200-M; Carl Zeiss, Göttingen, Germany) equipped with an ApoTome slider, which eliminates image blurring. Image processing was performed using an AxioCam MR chargecoupled device camera and AxioVision software (Carl Zeiss). Immunohistochemistry was performed using a Bond Max automated immunohistochemistry staining system (Leica, Nanterre, France), according to the manufacturer's protocol. After antigen retrieval in citrate buffer, the following antibodies were used: anti-GFP antibody (1:400, Invitrogen, A-11122); anti-CEACAM6 antibody (clone 9A6, 1:100, Abcam, Paris, France); anti-CD68 antibody (1:200, Dako); M30 cytodeath antibody (1:200, Roche Diagnostics, Meylan, France) which recognizes a cytokeratin-18 neoepitope that becomes available at an early caspase cleavage event during apoptosis and is considered an early marker of apoptosis in epithelial cells; ${ }^{36,37}$ cleaved caspase-3 antibody (1:800; Cell Signaling 9661, Danvers, MA); anti-NFkBp65 antibody (1:300, Santa Cruz, CA, SC-8008). Diaminobenzidine (DAB) was used as a chromogen. A light nuclear counterstaining was performed with hematoxylin. The percentage of nuclear staining of intestinal epithelial cells for NFKB p65 was assessed by counting the nuclei of epithelial cells in well-shaped colonic crypts, on 10 consecutive microscopic fields at a $\times 400$ magnification. The percentage of colonic crypts containing at least $30 \%$ caspase-3-positive epithelial cells was assessed by counting a total of about 100 well-oriented crypts at a $\times 200$ magnification.

Caspase-1 activity assay. After the $4 \mathrm{~h}$ incubation of mucosa explant cultures with bacteria (LF82 or $S$. Typhimurium), mucosa explants were lysed for $1 \mathrm{~h}$ on ice, centrifuged at $12,000 \mathrm{~g}$ for $20 \mathrm{~min}$. Caspase- 1 activity was assessed in the lysates using the Caspase-1 Colorimetric Assay Kit (Biovision, Milpitas, CA) according to the manufacturer's instructions. In brief, $200 \mu \mathrm{g}$ of total protein (determined in the lysates by the Biorad DC protein assay kit) was incubated with the colorimetric substrate for caspase-1 (YVAD-pNA) for $2 \mathrm{~h}$ at $37^{\circ} \mathrm{C}$ in a 96 -well plate. The absorbance was measured at a wavelength of $405 \mathrm{~nm}$ in a spectrofluorimeter (Victor 3, Perkin Elmer, Courtaboeuf, France).
Immunoblot analysis. For total protein extraction, explant cultures were homogenized in a stringent SDS-containing RIPA buffer as previously described. ${ }^{38}$ Samples were loaded on polyacrylamide gels (ANY KD Mini Protean gels, Bio-Rad). Proteins were electrotransferred onto nitrocellulose membranes (Bio-Rad, Marnes-laCoquette, France). After blocking, the membranes were probed with mouse monoclonal antibodies directed to Phospho-IкB $\alpha$ (ser32/36) (1:1,000, Cell Signaling 9246) or to $\beta$-actin $(1: 20,000$, clone AC-15, Sigma), or rabbit polyclonal antibodies directed to cleaved caspase-3 (1:1,000, Cell Signaling 9661), followed by the corresponding horseradish peroxidase-conjugated antibodies (anti-mouse 1:2,000; Santa Cruz, or anti-rabbit 1:20,000; Jackson ImmunoResearch, Newmarket, UK). The immunoreactive proteins were detected on films using an enhanced chemiluminescence substrate according to the manufacturer's instructions (Roche Diagnostics).

Cytokine determination assay. The following cytokines were determined by ELISA in samples of culture medium collected at the end of experiments, according to the manufacturers' protocols: IL-18 (Bender MedSystems; eBiosciences, San Diego, CA) and TNF $\alpha$ (PeproTech, Neuilly, France). Results are expressed as $\mathrm{pg} \mathrm{ml}^{-1}$.

Statistics. Each experiment was performed at least in triplicate, with at least three explant cultures for each condition. Statistical analyses were performed with GraphPad Prism (GraphPad Software, La Jolla, CA) using the Mann-Whitney $U$ test. A $P$-value less than 0.05 was considered significant.

SUPPLEMENTARY MATERIAL is linked to the online version of the paper at http://www.nature.com/mi

\section{ACKNOWLEDGMENTS}

We thank Pr Arlette Darfeuille-Michaud for providing the LF82 and LF82GFP strains. We thank Michèle Micheneau, Stéphanie Blandin and Cécile Deleine for expert technical assistance, Philippe Hulin from the Cellular Imaging Core Facility (MicroPICell, SFR François Bonamy, Nantes), and Nicolas Leboeuf from the "Photologie" Department for his help. We also thank Dr Anne-Françoise Miegeville for her help. EA 4273 and EA 3826 are funded by the Ministère de la Recherche. CNRS UMR 8076 is funded by CNRS and University Paris-Sud. A.J. is the recipient of a tenure position supported by INSERM.

\section{DISCLOSURE}

The authors declare no conflict of interest.

c) 2015 Society for Mucosal Immunology

\section{REFERENCES}

1. Martinon, F., Mayor, A. \& Tschopp, J. The inflammasomes: guardians of the body. Annu. Rev. Immunol. 27, 229-265 (2009).

2. Jarry, A. et al. Interleukin 1 and interleukin 1beta converting enzyme (caspase 1) expression in the human colonic epithelial barrier. Caspase 1 downregulation in colon cancer. Gut 45, 246-251 (1999).

3. Pages, F. et al. Modulation of interleukin-18 expression in human colon carcinoma: consequences for tumor immune surveillance. Int. J. Cancer 84, 326-330 (1999).

4. McAlindon, M.E., Hawkey, C.J. \& Mahida, Y.R. Expression of interleukin 1 beta and interleukin 1 beta converting enzyme by intestinal macrophages in health and inflammatory bowel disease. Gut 42, 214-219 (1998).

5. Jarry, A., Bossard, C., Sarrabayrouse, G., Mosnier, J.-F. \& Laboisse, C.L. Loss of interleukin-10 or transforming growth factor beta signaling in the human colon initiates a T-helper 1 response via distinct pathways. Gastroenterology 141, 1887-1896. e1881-1882 (2011).

6. Fink, S.L. \& Cookson, B.T. Pyroptosis and host cell death responses during Salmonella infection. Cell Microbiol. 9, 2562-2570 (2007).

7. Miao, E.A. et al. Caspase-1-induced pyroptosis is an innate immune effector mechanism against intracellular bacteria. Nat. Immunol. 11, 1136-1142 (2010). 
8. Hayden, M.S. \& Ghosh, S. Signaling to NF-kappaB. Gene. Dev. 18, 2195-2224 (2004).

9. Sadikot, R.T. et al. Targeted immunomodulation of the NF-kappaB pathway in airway epithelium impacts host defense against $P$ seudomonas aeruginosa. J. Immunol. 176, 4923-4930 (2006).

10. Jiang, D. et al. Airway epithelial NF-kappaB activation promotes Mycoplasma pneumoniae clearance in mice. PloS One 7, e52969 (2012).

11. Jobin, C. \& Sartor, R.B. The I kappa B/NF-kappa B system: a key determinant of mucosal inflammation and protection. Am. J. Physiol. Cell Physiol. 278, C451-C462 (2000).

12. Karin, M. \& Lin, A. NF-kappaB at the crossroads of life and death. Nat. Immunol. 3, 221-227 (2002).

13. Jarry, A. et al. Mucosal IL-10 and TGF-beta play crucial roles in preventing LPS-driven, IFN-gamma-mediated epithelial damage in human colon explants. J. Clin. Invest. 118, 1132-1142 (2008).

14. Di Sabatino, A. et al. Blockade of transforming growth factor beta upregulates T-box transcription factor T-bet, and increases $\mathrm{T}$ helper cell type 1 cytokine and matrix metalloproteinase-3 production in the human gut mucosa. Gut 57, 605-612 (2008).

15. Croxen, M.A. et al. Recent advances in understanding enteric pathogenic Escherichia coli. Clin. Microbiol. Rev. 26, 822-880 (2013).

16. Darfeuille-Michaud, A. et al. High prevalence of adherent-invasive Escherichia coli associated with ileal mucosa in Crohn's disease. Gastroenterology 127, 412-421 (2004).

17. Pineton de Chambrun, G., Colombel, J.-F., Poulain, D. \& DarfeuilleMichaud, A. Pathogenic agents in inflammatory bowel diseases. Curr. Opin. Gastroenterol. 24, 440-447 (2008).

18. Darfeuille-Michaud, A. et al. Presence of adherent Escherichia coli strains in ileal mucosa of patients with Crohn's disease. Gastroenterology 115, 1405-1413 (1998).

19. Jensen, S.R., Fink, L.N., Nielsen, O.H., Brynskov, J. \& Brix, S. Ex vivo intestinal adhesion of Escherichia coli LF82 in Crohn's disease. Microb. Pathog. 51, 426-431 (2011).

20. Boudeau, J., Glasser, A.L., Masseret, E., Joly, B. \& Darfeuille-Michaud, A. Invasive ability of an Escherichia coli strain isolated from the ileal mucosa of a patient with Crohn's disease. Infect. Immun. 67, 4499-4509 (1999).

21. Glasser, A.L. et al. Adherent invasive Escherichia coli strains from patients with Crohn's disease survive and replicate within macrophages without inducing host cell death. Infect. Immun. 69, 5529-5537 (2001).

22. Eaves-Pyles, T. et al. Escherichia coli isolated from a Crohn's disease patient adheres, invades, and induces inflammatory responses in polarized intestinal epithelial cells. Int. J. Med. Microbiol. 298, 397-409 (2008).

23. Bringer, M.A., Billard, E., Glasser, A.L., Colombel, J.-F. \& Darfeuille-Michaud, A. Replication of Crohn's disease-associated AIEC within macro- phages is dependent on TNF-alpha secretion. Lab Invest 92, 411-419 (2012).

24. Dunne, K.A. et al. Increased S-nitrosylation and proteasomal degradation of caspase-3 during infection contribute to the persistence of adherent invasive Escherichia coli (AIEC) in immune cells. PloS One 8, e68386 (2013).

25. Albina, J.E. On the expression of nitric oxide synthase by human macrophages. Why no NO?. J. Leuk. Biol. 58, 643-649 (1995).

26. Barnich, N. et al. CEACAM6 acts as a receptor for adherent-invasive E. coli, supporting ileal mucosa colonization in Crohn disease. J. Clin. Invest. 117, 1566-1574 (2007).

27. Grassl, G.A. \& Finlay, B.B. Pathogenesis of enteric Salmonella infections. Curr. Opin. Gastroenterol. 24, 22-26 (2008).

28. Lievin-Le Moal, V. \& Servin, A.L. Pathogenesis of human enterovirulent bacteria: lessons from cultured, fully differentiated human colon cancer cell lines. Microbiol. Mol. Biol. Rev. 77, 380-439 (2013).

29. Kumar, A., Takada, Y., Boriek, A.M. \& Aggarwal, B.B. Nuclear factorkappaB: its role in health and disease. J. Mol. Med. (Berl) 82, 434-448 (2004).

30. Karin, M. NF-kappaB and cancer: mechanisms and targets. Mol. Carcinog. 45, 355-361 (2006).

31. Tsilingiri, K. et al. Probiotic and postbiotic activity in health and disease: comparison on a novel polarised ex-vivo organ culture model. Gut 61, 1007-1015 (2012).

32. Hammarström, S. The carcinoembryonic antigen (CEA) family: structures, suggested functions and expression in normal and malignant tissues. Semin. Cancer Biol. 9, 67-81 (1999).

33. Coburn, B., Grassl, G.A. \& Finlay, B.B. Salmonella, the host and disease: a brief review. Immunol. Cell. Biol. 85, 112-118 (2007).

34. Barnich, N., Boudeau, J., Claret, L. \& Darfeuille-Michaud, A. Regulatory and functional co-operation of flagella and type 1 pili in adhesive and invasive abilities of AlEC strain LF82 isolated from a patient with Crohn's disease. Mol. Microbiol. 48, 781-794 (2003).

35. Cleynen, I. et al. Genetic and microbial factors modulating the ubiquitin proteasome system in inflammatory bowel disease. Gut 63, 1265-1274 (2014).

36. Caulin, C., Salvesen, G.S. \& Oshima, R.G. Caspase cleavage of keratin 18 and reorganization of intermediate filaments during epithelial cell apoptosis. J. Cell Biol. 138, 379-1394 (1997).

37. Leers, M.P. et al. Immunocytochemical detection and mapping of a cytokeratin 18 neo-epitope exposed during early apoptosis. J. Pathol. 187, 567-572 (1999).

38. Souaze, F. et al. Differential roles of Hath1, MUC2 and P27Kip1 in relation with gamma-secretase inhibition in human colonic carcinomas: a translational study. PloS One 8, e55904 (2013). 